Supporting information

for

\title{
Iterative Oxime Bond Chemistry Leads to Protease Inhibitors
}

Olivier Renaudet and Jean-Louis Reymond*

Spectroscopic data of active compounds $9, \mathbf{2 1}, \mathbf{3 5}$ and $\mathbf{3 8 - 4 0}$, protease assay conditions and basic crystallographic data for oligomers $\mathbf{1 9}$ and $\mathbf{4 1 .}$ 
2
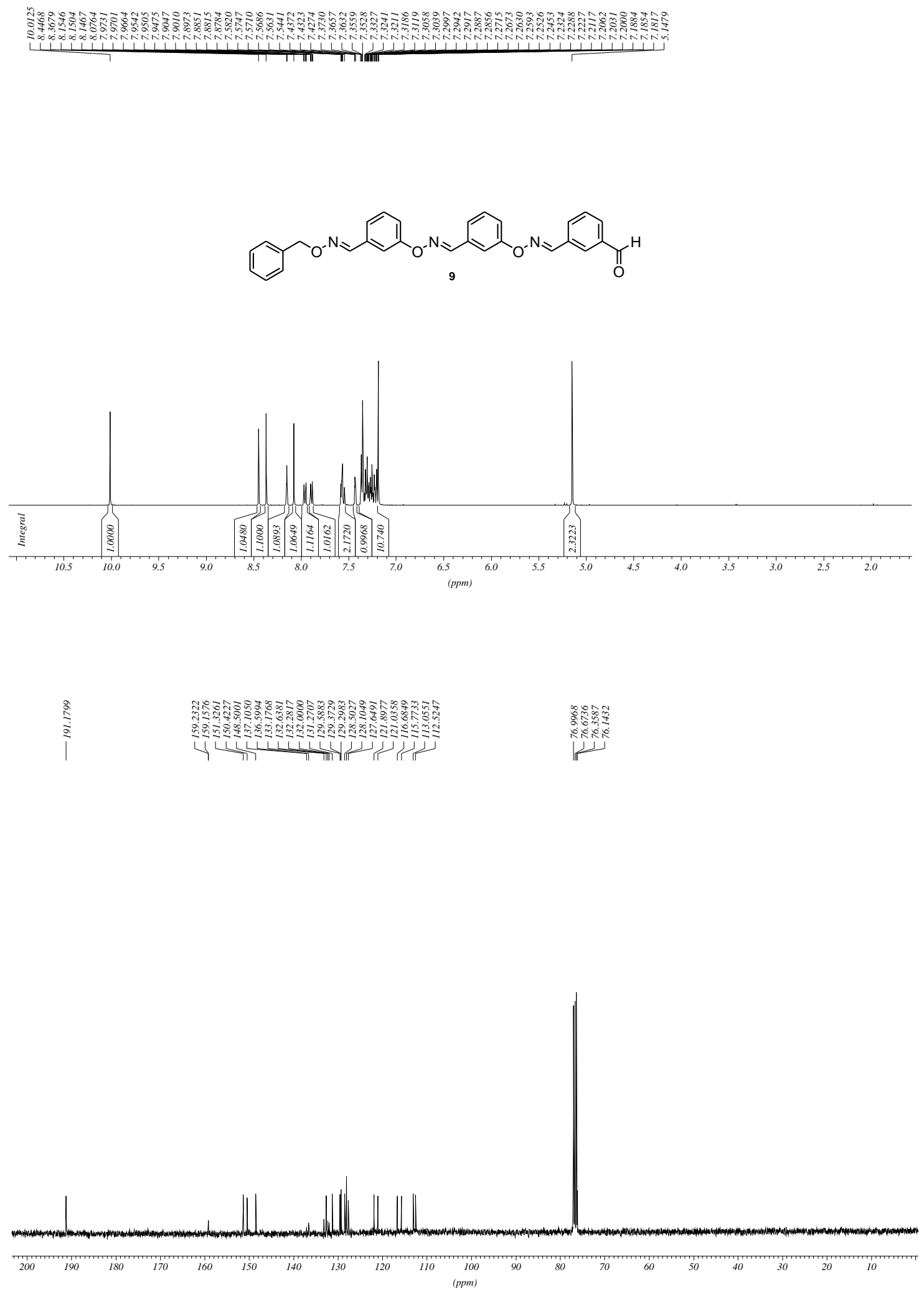

${ }^{1} \mathrm{H}$ NMR (400 MHz, $\left.\mathrm{CDCl}_{3}\right): \delta=10.01(\mathrm{~s}, 1 \mathrm{H}, \mathrm{CHO}), 8.44\left(\mathrm{~s}, 1 \mathrm{H}, \mathrm{H}_{\text {ox. }}\right), 8.37$ (s, $\left.1 \mathrm{H}, \mathrm{H}_{\text {ox. }}\right), 8.15$ (t, 1 $\left.\mathrm{H}, J=1.7 \mathrm{~Hz}, \mathrm{H}_{\text {ar. }}\right), 8.07\left(\mathrm{~s}, 1 \mathrm{H}, \mathrm{H}_{\text {ox. }}\right), 7.96\left(\mathrm{dt}, 1 \mathrm{H}, J=1.5,7.8 \mathrm{~Hz}, \mathrm{H}_{\text {ar. }}\right), 7.89(\mathrm{dt}, 1 \mathrm{H}, J=1.5,7.8$ $\left.\mathrm{Hz}, \mathrm{H}_{\text {ar. }}\right)$, 7.58-7.54 (m, $\left.2 \mathrm{H}, \mathrm{H}_{\text {ar. }}\right), 7.43$ (t, $\left.1 \mathrm{H}, J=1.9 \mathrm{~Hz}, \mathrm{H}_{\text {ar. }}\right), 7.37-7.18\left(\mathrm{~m}, 11 \mathrm{H}, \mathrm{H}_{\text {ar. }}\right), 5.15(\mathrm{~s}, 2 \mathrm{H}$, $\left.\mathrm{CH}_{2}\right) ;{ }^{13} \mathrm{C}$ NMR $\left(100 \mathrm{MHz}, \mathrm{CDCl}_{3}\right): \delta=191.2,159.2,159.1,151.3,150.4,148.5,137.1,136.6,133.2$, 132.6, 132.3, 132.0, 131.3, 129.6, 129.4, 129.3, 128.5, 128.1, 127.6, 122.1, 121.9, 121.0, 116.7, 115.8, 113.0, 112.5, 76.1; HREI-MS: calcd. for $\mathrm{C}_{29} \mathrm{H}_{24} \mathrm{~N}_{3} \mathrm{O}_{4}$ : 478.1766; found: 478.1779 [M+H] ${ }^{+}$. 

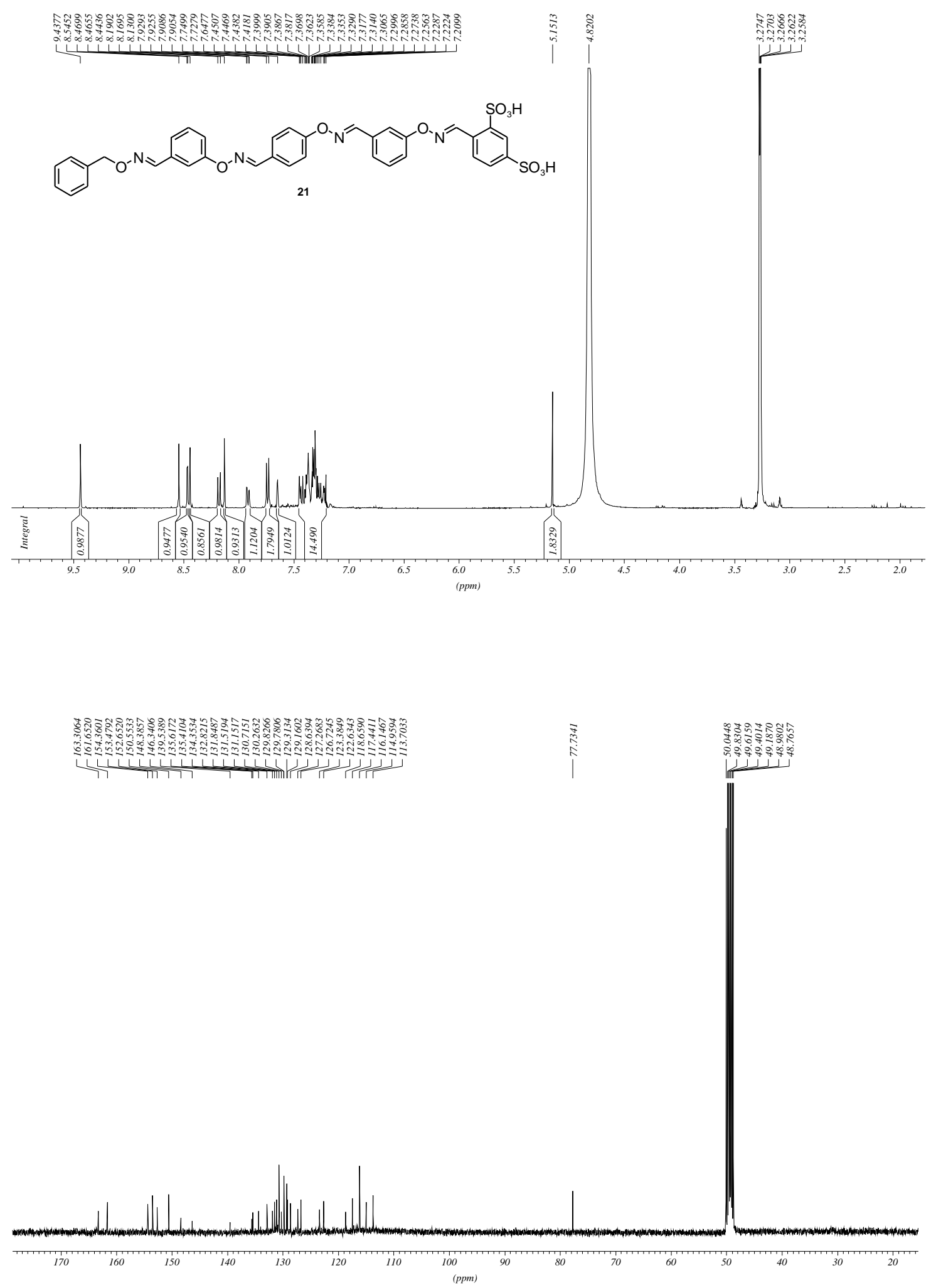

${ }^{1} \mathrm{H}$ NMR (400 MHz, $\left.\mathrm{CD}_{3} \mathrm{OD}\right): \delta=9.43$ (s, $1 \mathrm{H}, \mathrm{H}_{\text {ox. }}$ ), $8.54\left(\mathrm{~s}, 1 \mathrm{H}, \mathrm{H}_{\text {ox. }}\right), 8.46\left(\mathrm{~d}, 1 \mathrm{H}, J=1.8 \mathrm{~Hz}, \mathrm{H}_{\text {ar. }}\right.$ ), $8.44\left(\mathrm{~s}, 1 \mathrm{H}, \mathrm{H}_{\mathrm{ox}}\right), 8.17\left(\mathrm{~d}, 1 \mathrm{H}, J=8.3 \mathrm{~Hz}, \mathrm{H}_{\mathrm{arr}}\right), 8.13\left(\mathrm{~s}, 1 \mathrm{H}, \mathrm{H}_{\mathrm{ox}}\right), 7.91(\mathrm{dd}, 1 \mathrm{H}, J=1.4,8.3 \mathrm{~Hz}$, $\left.\mathrm{H}_{\text {ar. }}\right)$, 7.75-7.64 (m, $\left.3 \mathrm{H}, \mathrm{H}_{\text {ar. }}\right), 7.45-7.20\left(\mathrm{~m}, 14 \mathrm{H}, \mathrm{H}_{\mathrm{ar} .}\right), 5.15\left(\mathrm{~s}, 2 \mathrm{H}, \mathrm{CH}_{2}\right) ;{ }^{13} \mathrm{C} \mathrm{NMR}(100 \mathrm{MHz}$, $\left.\mathrm{CD}_{3} \mathrm{OD}\right): \delta=163.3,161.6,154.4,153.5,152.7,150.6,148.4,146.3,139.5,135.6,135.4,134.4,132.8$, $131.8,131.5,131.2,130.7,130.3,129.8,129.7,129.3,129.1,128.6,127.3,126.7,123.4,122.6,118.7$, 117.4, 116.1, 114.9, 113.7, 77.7; EI-MS: calcd. for $\mathrm{C}_{35} \mathrm{H}_{28} \mathrm{~N}_{4} \mathrm{O}_{10} \mathrm{~S}_{2}$ : 728.12 ; found: $728.89[\mathrm{M}+\mathrm{H}]^{+}$. 

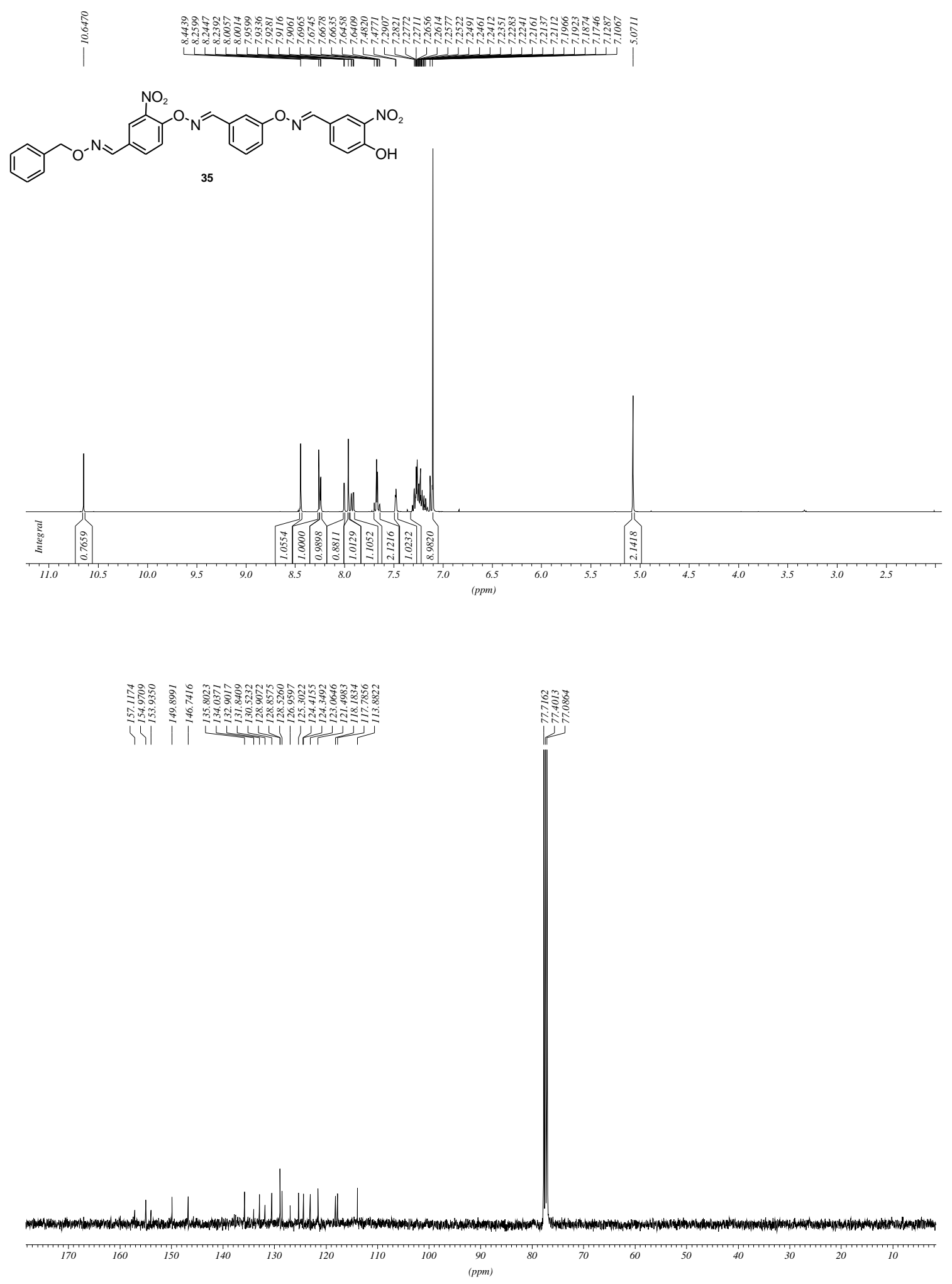

${ }^{1} \mathrm{H}$ NMR (400 MHz, $\left.\mathrm{CDCl}_{3}\right): \delta=10.64(\mathrm{~s}, 1 \mathrm{H}, \mathrm{OH}), 8.44\left(\mathrm{~s}, 1 \mathrm{H}, \mathrm{H}_{\text {ox. }}\right), 8.26\left(\mathrm{~s}, 1 \mathrm{H}, \mathrm{H}_{\text {ox. }}\right), 8.24(\mathrm{~d}, 1$ $\left.\mathrm{H}, J=2.2 \mathrm{~Hz}, \mathrm{H}_{\text {ar. }}\right), 8.00\left(\mathrm{~d}, 1 \mathrm{H}, J=1.7 \mathrm{~Hz}, \mathrm{H}_{\mathrm{arr}}\right), 7.96\left(\mathrm{~s}, 1 \mathrm{H}, \mathrm{H}_{\mathrm{ox} .}\right), 7.91(\mathrm{dd}, 1 \mathrm{H}, J=2.2,8.8 \mathrm{~Hz}$, $\mathrm{H}_{\text {ar. }}$ ) 7.69-7.64 (m, $\left.2 \mathrm{H}, \mathrm{H}_{\text {ar. }}\right), 7.48-7.46\left(\mathrm{~m}, 1 \mathrm{H}, \mathrm{H}_{\text {ar. }}\right)$, 7.30-7.10 (m, $\left.9 \mathrm{H}, \mathrm{H}_{\text {ar. }}\right), 5.07\left(\mathrm{~s}, 2 \mathrm{H}, \mathrm{CH}_{2}\right) ;{ }^{13} \mathrm{C}$ NMR $\left(100 \mathrm{MHz}, \mathrm{CDCl}_{3}\right): \delta=157.1,154.9,153.9,149.9,146.7,137.8,137.6,135.8,134.0,132.9$, $131.8,130.5,128.9,128.8,128.5,126.9,125.3,124.4,124.3,123.0,121.5,118.2,117.8,113.9,77.1$; HREI-MS: calcd. for $\mathrm{C}_{28} \mathrm{H}_{22} \mathrm{~N}_{5} \mathrm{O}_{8}$ : 556.1468; found: $556.1501[\mathrm{M}+\mathrm{H}]^{+}$. 

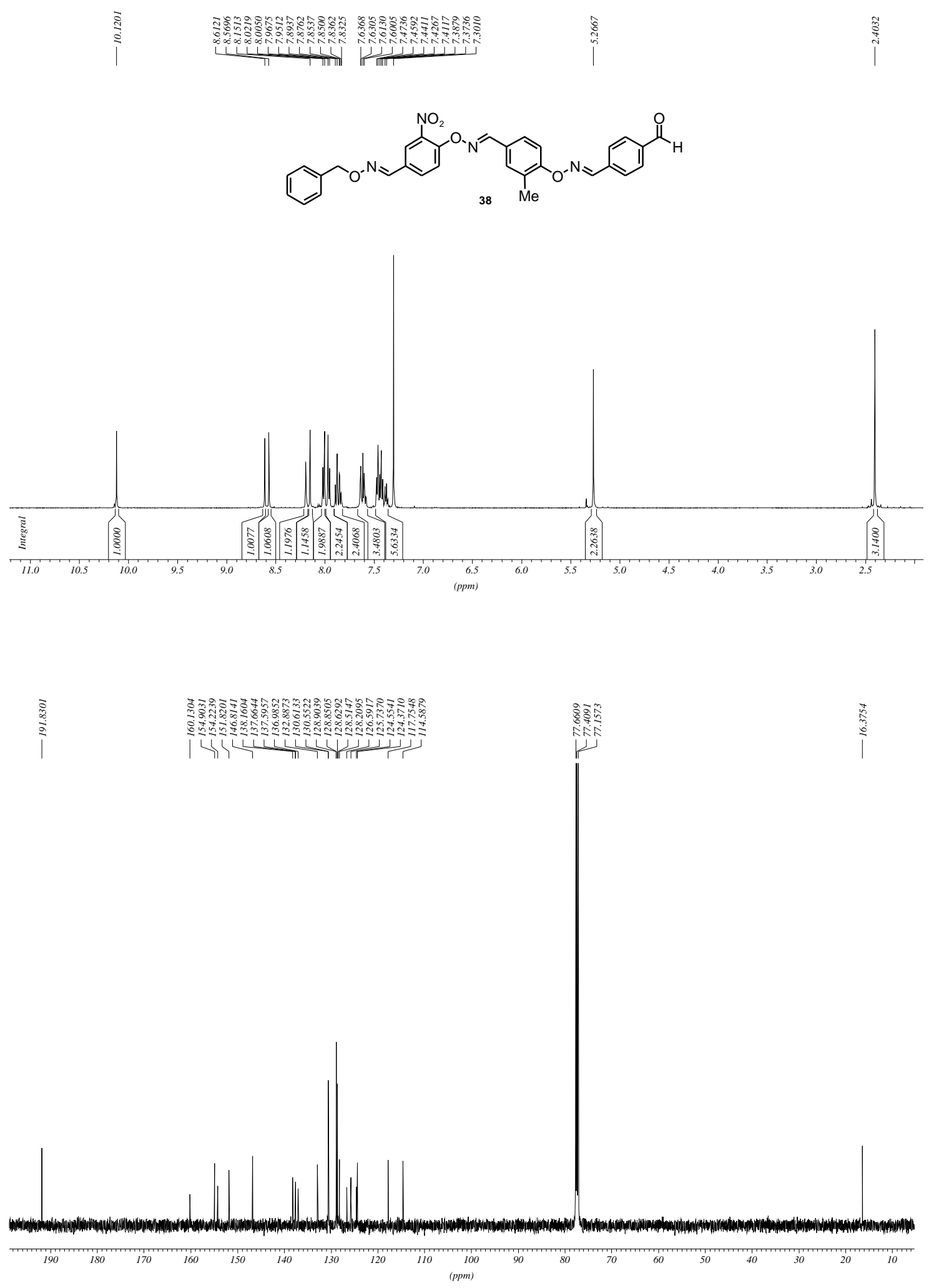

${ }^{1} \mathrm{H}$ NMR (400 MHz, $\mathrm{CDCl}_{3}$ ): $\delta=10.12$ (s, $\left.1 \mathrm{H}, \mathrm{CHO}\right), 8.61$ (s, $1 \mathrm{H}, \mathrm{H}_{\text {ox. }}$ ), 8.57 (s, $\left.1 \mathrm{H}, \mathrm{H}_{\text {ox. }}\right), 8.19$ (d, 1 $\left.\mathrm{H}, J=1.5 \mathrm{~Hz}, \mathrm{H}_{\text {ar. }}\right), 8.15\left(\mathrm{~s}, 1 \mathrm{H}, \mathrm{H}_{\mathrm{ox}}\right), 8.02-7.95\left(\mathrm{~m}, 4 \mathrm{H}, \mathrm{H}_{\text {ar }}\right), 7.89-7.83\left(\mathrm{~m}, 2 \mathrm{H}, \mathrm{H}_{\text {ar. }}\right), 7.63-7.58(\mathrm{~m}$, $\left.3 \mathrm{H}, \mathrm{H}_{\text {ar }}\right), 7.47-7.35\left(\mathrm{~m}, 5 \mathrm{H}, \mathrm{H}_{\text {ar. }}\right), 5.26\left(\mathrm{~s}, 2 \mathrm{H}, \mathrm{CH}_{2}\right), 2.40\left(\mathrm{~s}, 3 \mathrm{H}, \mathrm{CH}_{3}\right) ;{ }^{13} \mathrm{C} \mathrm{NMR}(100 \mathrm{MHz}$, $\left.\mathrm{CDCl}_{3}\right): \delta=191.8,160.1,154.9,154.2,151.8,146.8,138.1,137.6,137.5,136.9,132.9,130.6,130.5$, 128.9, 128.8, 128.6, 128.5, 128.2, 126.6, 125.7, 124.5, 124.4, 117.7, 114.6, 76.9, 16.4; HREI-MS: calcd. for $\mathrm{C}_{30} \mathrm{H}_{25} \mathrm{~N}_{4} \mathrm{O}_{6}$ : 537.1774; found: $537.1806[\mathrm{M}+\mathrm{H}]^{+}$. 

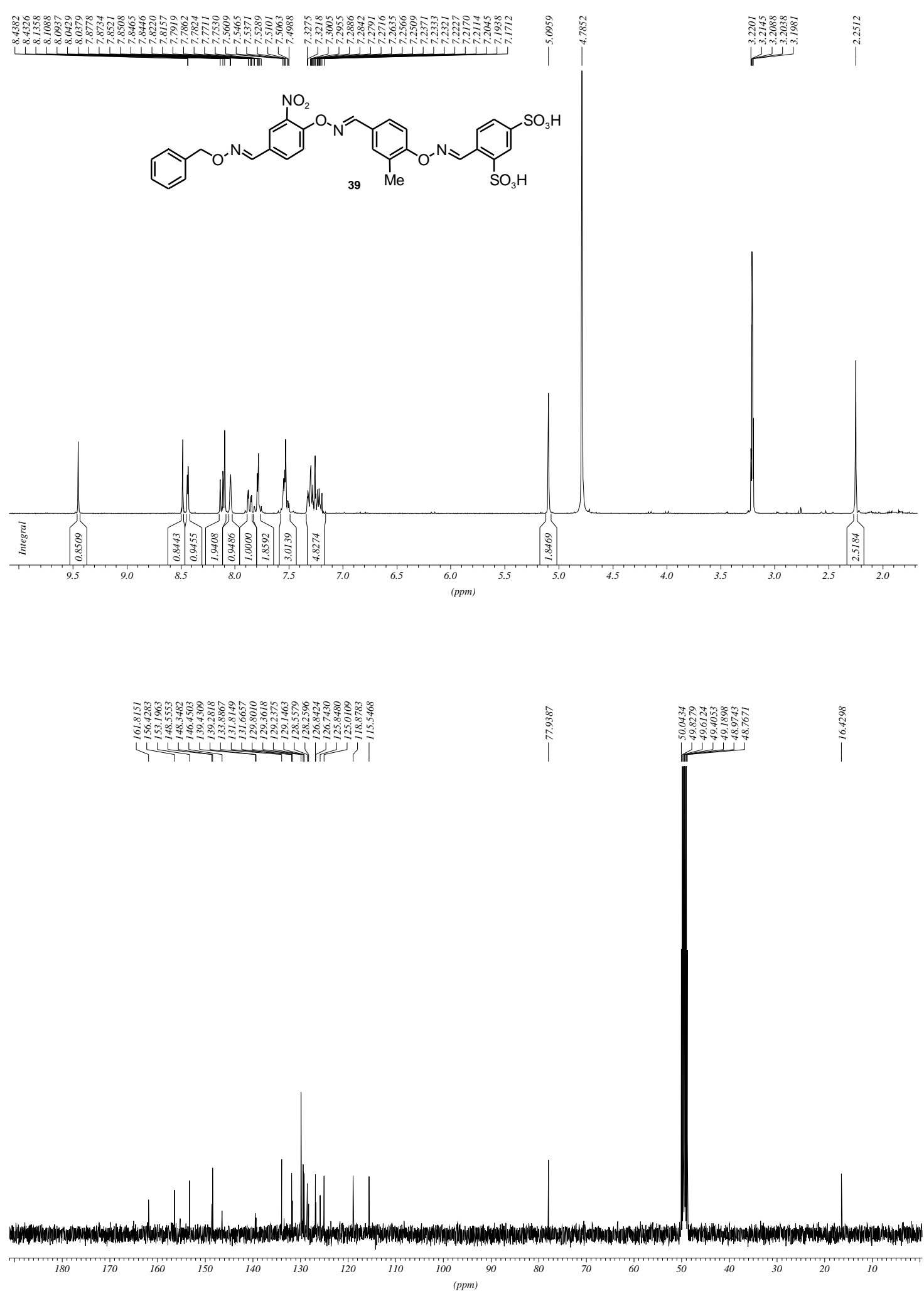

${ }^{1} \mathrm{H}$ NMR (400 MHz, CD $\left.\mathrm{CD}_{3} \mathrm{OD}\right): \delta=9.45\left(\mathrm{~s}, 1 \mathrm{H}, \mathrm{H}_{\text {ox. }}\right), 8.48\left(\mathrm{~s}, 1 \mathrm{H}, \mathrm{H}_{\text {ox. }}\right), 8.43\left(\mathrm{~d}, 1 \mathrm{H}, J=1.7 \mathrm{~Hz}, \mathrm{H}_{\text {ar. }}\right)$, $8.11\left(\mathrm{~d}, 1 \mathrm{H}, J=8.1 \mathrm{~Hz}, \mathrm{H}_{\mathrm{ar}}\right), 8.09$ (s, $\left.1 \mathrm{H}, \mathrm{H}_{\text {ox. }}\right), 8.04\left(\mathrm{~d}, 1 \mathrm{H}, J=1.5 \mathrm{~Hz}, \mathrm{H}_{\mathrm{ar}}\right.$ ), 7.86 (dd, $1 \mathrm{H}, J=1.3$, 8.1 Hz, $\left.\mathrm{H}_{\text {ar }}\right), 7.82-7.77\left(\mathrm{~m}, 2 \mathrm{H}, \mathrm{H}_{\mathrm{ar}}\right), 7.56-7.49\left(\mathrm{~m}, 3 \mathrm{H}, \mathrm{H}_{\mathrm{ar} .}\right), 7.32-7.17\left(\mathrm{~m}, 5 \mathrm{H}, \mathrm{H}_{\text {ar }}\right), 5.09(\mathrm{~s}, 2 \mathrm{H}$, $\left.\mathrm{CH}_{2}\right) ;{ }^{13} \mathrm{C}$ NMR $\left(100 \mathrm{MHz}, \mathrm{CD}_{3} \mathrm{OD}\right): \delta=161.8,156.4,153.2,148.6,148.3,146.5,139.4,139.3$, 133.9, 131.8, 131.7, 129.8, 129.4, 129.2, 129.1, 128.6, 128.3, 126.8, 126.7, 125.8, 125.0, 118.9, 115.5, 77.9, 16.4; EI-MS: calcd. for $\mathrm{C}_{29} \mathrm{H}_{25} \mathrm{~N}_{4} \mathrm{O}_{11} \mathrm{~S}_{2}$ : 669.0961; found: 669.0967 [M+H] . 

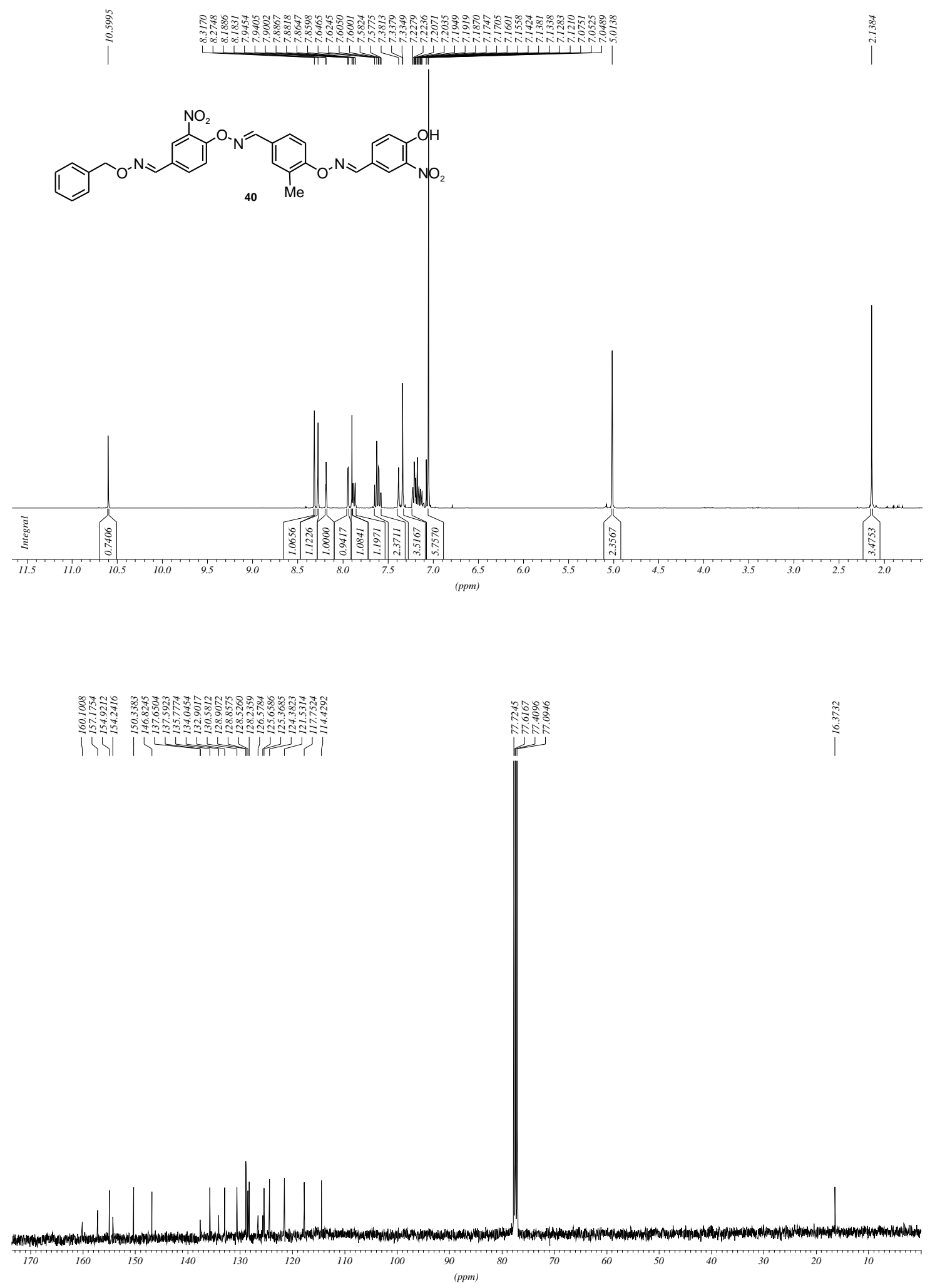

${ }^{1} \mathrm{H}$ NMR (400 MHz, $\mathrm{CDCl}_{3}$ ): $\delta=10.60$ (s, $\left.1 \mathrm{H}, \mathrm{OH}\right), 8.31$ (s, $1 \mathrm{H}, \mathrm{H}_{\text {ox. }}$ ), 8.27 (s, $1 \mathrm{H}, \mathrm{H}_{\text {ox. }}$ ), 8.18 (d, 1 $\left.\mathrm{H}, J=2.2 \mathrm{~Hz}, \mathrm{H}_{\text {ar }}\right), 7.94\left(\mathrm{~d}, 1 \mathrm{H}, J=1.9 \mathrm{~Hz}, \mathrm{H}_{\text {ar. }}\right), 7.90\left(\mathrm{~s}, 1 \mathrm{H}, \mathrm{H}_{\mathrm{ox}}\right), 7.87(\mathrm{dd}, 1 \mathrm{H}, J=1.9,8.8 \mathrm{~Hz}$, $\mathrm{H}_{\text {ar. }}$ ), 7.64-7.57 (m, $\left.2 \mathrm{H}, \mathrm{H}_{\text {ar. }}\right), 7.38-7.33\left(\mathrm{~m}, 3 \mathrm{H}, \mathrm{H}_{\text {ar. }}\right), 7.22-7.07\left(\mathrm{~m}, 6 \mathrm{H}, \mathrm{H}_{\mathrm{ar}}\right), 5.01\left(\mathrm{~s}, 2 \mathrm{H}, \mathrm{CH}_{2}\right)$, $2.14\left(\mathrm{~s}, 3 \mathrm{H}, \mathrm{CH}_{3}\right) ;{ }^{13} \mathrm{C}$ NMR $\left(100 \mathrm{MHz}, \mathrm{CDCl}_{3}\right): \delta=160.1,157.1,154.9,154.2,150.3,146.8,137.6$, 137.6, 135.8, 134.0, 132.9, 130.6, 128.9, 128.8, 128.5, 128.2, 126.6, 125.6, 125.4, 124.4, 124.4, 121.5, $117.7,115.6,114.4,77.6,16.4$. 


\section{Inhibition data for $\alpha$-chymotrypsin:}

Assay conditions: $5 \mu \mathrm{g} . \mathrm{mL}^{-1} \alpha$-Chymotrypsin (from Bovine Pancreas, Sigma C-7762), 500 $\mu \mathrm{M} N$-succinyl-Ala-Ala-Pro-Phe p-nitroanilide (Sigma S-7388), $5 \mathrm{mM}$ aq. Bis Tris, $\mathrm{pH} 7.2$, $26{ }^{\circ} \mathrm{C} .40 \mu \mathrm{L}$ assays were run in half-area clear polystyrene 96-well plates (Costar). Release of 4-nitroaniline was followed spectroscopically at $405 \mathrm{~nm}$ using a microtiter-plate reader. Inhibition of proteolytic activity was studied in the presence of $10 \mu \mathrm{M}$ inhibitor for screening the oxime oligomer library. $\mathrm{IC}_{50}$ values of most active compounds (compounds with more than $90 \%$ inhibition) were measured under the same conditions in the presence of different concentration of inhibitor. 


\section{Basic crystallographic data for oligomer 19:}

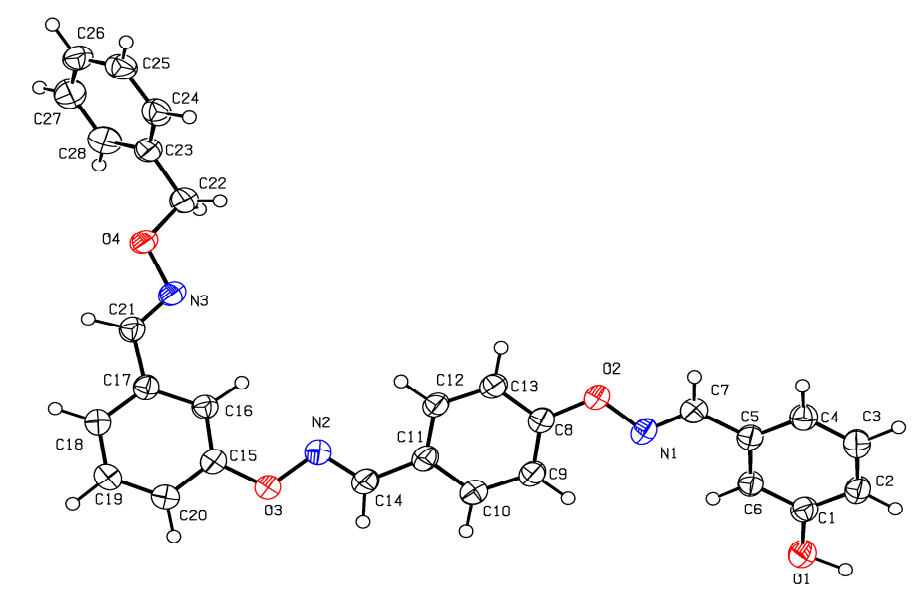

\section{Identification code \\ Crystal shape \\ Crystal colour \\ Crystal size \\ Empirical formula \\ Formula weight \\ Crystal system \\ Space group \\ Unit cell dimensions}

Volume

Cell refinement parameters

Reflections

Angle range

Density (calculated)

Radiation used

Wavelength

Linear absorption coefficient

Temperature

\section{9}

plate

colourless

$0.40 \times 0.40 \times 0.10 \mathrm{~mm}$

C28 H23 N3 O4

465.49

Monoclinic

P 2 / / C

$\mathrm{a}=10.1436(11) \mathrm{A}$ alpha $=90 \mathrm{deg}$.

$\mathrm{b}=8.0346(6) \mathrm{A} \quad$ beta $=96.601(10)$ deg.

$\mathrm{c}=29.122(4) \mathrm{A}$ gamma $=90 \mathrm{deg}$.

$2357.7(4) \quad A^{\wedge} 3$

16444

$2.02<$ thet $a<29.63$

4

$1.311 \mathrm{~g} / \mathrm{cm}^{\wedge} 3$

MoK\a

$0.71073 \mathrm{~A}$

$0.089 \mathrm{~mm}^{\wedge}-1$

$153(2) \mathrm{K}$ 


\section{Basic crystallographic data for oligomer 41:}

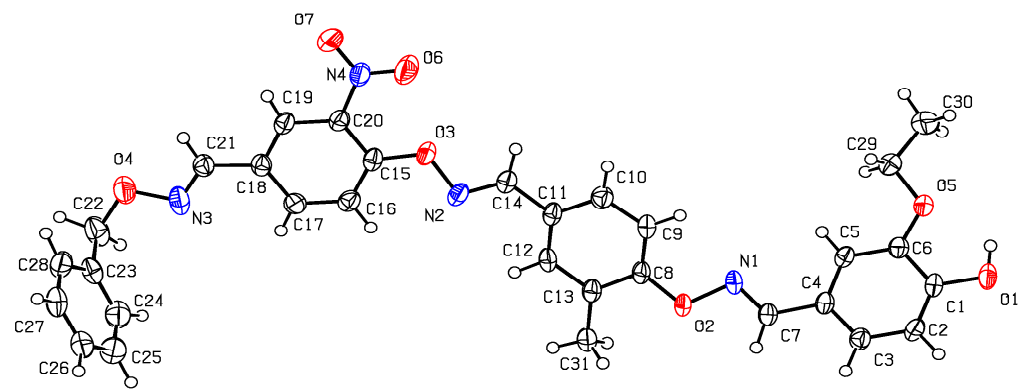

Identification code
Crystal shape
Crystal colour
Crystal size
Empirical formula
Formula weight
Crystal system
Space group
Unit cell dimensions

Volume

Cell refinement parameters

Reflections

Angle range

Z

Density (calculated)

Radiation used

Wavelength

Linear absorption coefficient

\section{1}

plate

yellow

$0.35 \times 0.35 \times 0.10 \mathrm{~mm}$

C31 H28 N4 O7

568.57

Triclinic

$\mathrm{P}-1$

$\mathrm{a}=10.2388(8)$ A alpha $=85.466(10) \mathrm{deg}$.

$\mathrm{b}=11.3897(10) \mathrm{A}$ beta $=73.957(9)$ deg.

$\mathrm{c}=12.7626(10)$ A gamma $=76.834(10) \mathrm{deg}$.

$1392.6(2) A^{\wedge} 3$

6472

$2.12<$ theta $<25.940$

2

$1.356 \mathrm{~g} / \mathrm{cm}^{\wedge} 3$

MoK \a

$0.71073 \mathrm{~A}$

$0.098 \mathrm{~mm}^{\wedge}-1$

$153(2) \mathrm{K}$ 